\title{
Near-infrared photometry of isolated spirals with and without an AGN
}

\author{
I. The $\operatorname{data}^{\star, \star \star}$ \\ I. Márquez ${ }^{1,2}$, F. Durret ${ }^{2,3}$, R.M. González Delgado ${ }^{1}$, I. Marrero ${ }^{1}, J$. Masegosa ${ }^{1}, J_{.}$Maza $^{4}$, M. Moles $^{5}$, E. Pérez $^{1}$, \\ and M. Roth ${ }^{6}$ \\ 1 Instituto de Astrofísica de Andalucía (CSIC), Apartado 3004, E-18080 Granada, Spain \\ 2 Institut d'Astrophysique de Paris, CNRS, 98bis Bd. Arago, F-75014 Paris, France \\ 3 DAEC, Observatoire de Paris, Université Paris VII, CNRS (UA 173), F-92195 Meudon Cedex, France \\ 4 Departamento de Astronomía, Universidad de Chile, Casilla 36D, Santiago, Chile \\ ${ }^{5}$ Instituto de Matemáticas y Física Fundamental (CSIC), Madrid, Spain and Observatorio Astronómico Nacional, Madrid, \\ Spain. Presently on sabbatical leave at Queen Mary \& Westfield College, London, UK \\ ${ }^{6}$ Observatories of the Carnegie Institution of Washington, 813 Barbara Street, Pasadena, CA 91101, U.S.A.
}

Received May 5; accepted August 27, 1999

\begin{abstract}
We present infrared imaging data in the $J$ and $K^{\prime}$ bands obtained for 18 active spiral galaxies, together with 11 non active galaxies taken as a control sample. All of them were chosen to satisfy well defined isolation criteria so that the observed properties are not related to gravitational interaction. For each object we give: the image in the $K^{\prime}$ band, the sharp-divided image (obtained by dividing the observed image by a filtered one), the difference image (obtained by subtracting a model to the observed one), the color $J-K^{\prime}$ image, the ellipticity and position angle profiles, the surface brightness profiles in $J$ and $K^{\prime}$, their fits by bulge+disk models and the color gradient.

We have found that four (one) active (control) galaxies previously classified as non-barred turn out to have bars when observed in the near-infrared. One of these four galaxies (UGC 1395) also harbours a secondary bar. For 15 (9 active, 6 control) out of 24 (14 active, 10 control) of the optically classified barred galaxies (SB or SX) we find that a secondary bar (or a disk, a lense or an elongated ring) is present.
\end{abstract}

The work presented here is part of a large program (DEGAS) aimed at finding out whether there are differ-

Send offprint requests to: I. Márquez (isabel@iaa.es)

* Based on data obtained at: the European Southern Observatory, La Silla, Chile, the Télescope Bernard Lyot, Calar Alto Observatory, Las Campanas Observatory. Also based on observations made with the NASA/ESA Hubble Space Telescope, obtained from the data archive at the Space Telescope Institute.

** Figures 1-35 are only available in electronic form at the http://www.edpsciences.org ences between active and non active galaxies in the properties of their central regions that could be connected with the onset of nuclear activity.

Key words: galaxies: active — galaxies: fundamental parameters — galaxies: photometry — infrared: galaxies

\section{Introduction}

The existence of non-axysimmetric components of the galactic potential has been frequently invoked as an efficient way to transport gas from the galaxy scale down to the nucleus to fuel the AGN. In particular, the shocks and gravitational torques induced by a galactic bar ${ }^{1}$ can make the gas loose angular momentum and therefore facilitate the fuelling mechanism (Shlosman et al. 1989). Nevertheless, it has been suggested that there is not a preference for Seyfert nuclei to occur in barred galaxies (Heckman 1980; Simkim et al. 1980). This result is confirmed by statistical analyses on the catalogued morphology of Seyfert galaxies (Moles et al. 1995) and through near-infrared imaging studies (McLeod \& Rieke 1995; Alonso-Herrero et al. 1996; Mulchaey \& Regan 1997).

We have started a large observational program aimed at studying the conditions for the onset of activity in galactic nuclei. Based on previous work (Moles et al. 1995),

\footnotetext{
${ }^{1}$ We mean bar = primary bar; when considering secondary bars, it will be clearly stated.
} 
we search for detailed morphological and kinematical differences between active and non active galaxies of similar global morphology. We particularly pay attention to those that could facilitate the transport of gas towards the very central regions and the nucleus (for an example of the complete analysis of one of our sample galaxies see Pérez et al. 1999). For this purpose, we are obtaining optical and near-infrared images and long slit spectroscopy with the best possible spatial resolution.

We selected a sample of galaxies, out of which 18 host active nuclei and the rest form a control sample of non active galaxies (see Sect. 1).

In this paper we present the first data set: the infrared imaging data in the $J$ and $K^{\prime}$ bands. Infrared imaging is particularly important because it allows us to trace the old stellar population, and to separate the various components (the bulge, disk, bar(s) and spiral arms) with the smallest contribution of the active nucleus and less contamination by dust absorption. In fact, various $K$ band imaging studies have revealed that bars were present in galaxies classified as unbarred in the optical (McLeod \& Rieke 1995; Mulchaey \& Regan 1997), hence showing that the nearinfrared is better suited for these purposes. The sample is described in Sect. 2. In Sect. 3 we present the near-infrared data we have obtained together with the HST archive images we used. In Sect. 4 we describe the different methods of analysis applied to the images. In Sects. 5 and 6 the results are described for each of the galaxies in both sets of active and non-active spirals respectively. The summary is given in Sect. 7 .

A discussion on the NIR properties of these galaxies, together with the comparison of active versus non-active galaxies derived from these data will be presented in a companion paper (Márquez et al., in preparation).

\section{The sample}

The active galaxies have been chosen with the following criteria: (a) Seyfert 1 or 2 from the Véron-Cetty \& Véron (1993) catalogue; (b) with morphological information in the RC3 Catalogue; (c) isolated, in the sense of not having a companion within $0.4 \mathrm{Mpc}\left(H_{0}=75 \mathrm{~km} \mathrm{~s}^{-1} \mathrm{Mpc}\right)$ and $c z<500 \mathrm{~km} \mathrm{~s}^{-1}$, or companions catalogued by Nilson without known $z$; (d) nearby, $c z<6000 \mathrm{~km} \mathrm{~s}^{-1}$; and (e) intermediate inclination ( 30 to $65^{\circ}$ ). The control sample galaxies have been selected among spirals verifying the same conditions (b), (c), (d) and (e), and with types similar to those of the active spirals. Thus, all the galaxies in our sample are isolated, in the sense of avoiding possible effects of interactions with luminous nearby galaxies ${ }^{2}$.

The main properties of the sample galaxies, as given in the RC3 catalogue are presented in Table 1.

\footnotetext{
${ }^{2}$ In a strict sense, they are weakly interacting systems, since we don't take into account the possibility of being surrounded by dwarf galaxies or HI clouds.
}

The samples are not large enough to give statistically significant results, but the detailed study of each of the galaxies in these samples should give us at least hints on what differences between active and non-active isolated spiral galaxies, if any, are to be taken into account to identify the conditions for the onset of nuclear activity.

The active sample extends farther in redshift. In particular, there are six active galaxies with $c z>4000 \mathrm{~km}$ $\mathrm{s}^{-1}$, but none in the control sample. Although this changes the physical resolution achieved for those objects, we don't expect any bias due to that difference. Absolute mean $B$ luminosities give $M_{B}=-20.4 \pm 0.6$ for Seyferts and $M_{B}=-20.3 \pm 0.9$ for non-active galaxies. Some contribution from the active galaxies would be expected as a consequence of the presence of the active nucleus itself and of the higher levels of star formation sometimes observed in that kind of galaxy (Maiolino \& Rieke 1995). If any, this effect is not systematically present in our small samples, which can therefore be considered as comparable with respect to the host galaxy luminosity. The distribution of inclinations is almost flat for the non-active galaxies, whereas there is a deficiency of active galaxies at large inclinations, as expected for an optically selected sample of active galaxies (McLeod \& Rieke 1995). The morphological types of spiral active galaxies are earlier than Sbc, resembling the type distribution of active galaxies in the Véron-Cetty \& Véron catalogue (Moles et al. 1995); nonactive galaxies have been selected to have similar types. Looking at the presence of bars in the galaxies of both samples (as catalogued in the RC3), we notice that only one galaxy in the list of non-active objects is unbarred, and three more are of intermediate type X. For the active galaxies, half are barred and four more of type X. As already quoted, the presence of a primary bar does not seem to be the direct cause for the nuclear activity. Otherwise the lists of active and non active galaxies we have observed are rather similar in global properties. We therefore estimate that the sample of non-active galaxies is well suited to be used as a control sample.

\section{The data}

We have obtained $K^{\prime}$ images for the 18 active galaxies together with $J$ images for 15 of them. $J$ and $K^{\prime}$ images were taken for 11 non-active spirals. The infrared data have been acquired mainly with three telescopes: the $2 \mathrm{~m}$ Télescope Bernard Lyot (Pic du Midi Observatory, France), the $2.5 \mathrm{~m}$ DuPont telescope (Las Campanas, Chile), and the $3.5 \mathrm{~m}$ telescope at Calar Alto (Spain). We have also used some short observations taken with the ESO $2.2 \mathrm{~m}$ telescope in La Silla for the relative calibration of one of the $J$ images obtained during a non-photometric night. The journal of observations is presented in Table 2. We always used NICMOS-3 $256 \times 256$ detectors; the pixel scales are given in Table 2 . 
Table 1. Data for the sample galaxies in the RC3 catalogue

\begin{tabular}{|c|c|c|c|c|c|c|c|c|c|c|}
\hline $\begin{array}{l}\text { Name } \\
(1)\end{array}$ & $\begin{array}{r}\alpha(2000.0) \\
(2)\end{array}$ & $\begin{array}{r}\delta(2000.0) \\
(3)\end{array}$ & $\begin{array}{r}\text { PGC } \\
(4)\end{array}$ & $\begin{array}{l}B \\
(5)\end{array}$ & $\begin{array}{l}D_{25} \\
(6)\end{array}$ & $\begin{array}{l}R_{25} \\
(7)\end{array}$ & $\begin{array}{l}\text { PA } \\
(8)\end{array}$ & $\begin{array}{r}c z \\
(9)\end{array}$ & $\begin{array}{r}t \\
(10)\end{array}$ & $\begin{array}{r}\text { Seyfert } \\
(11)\end{array}$ \\
\hline \multicolumn{11}{|c|}{ Active galaxies } \\
\hline UGC 1395 & 015522.2 & +063641 & 7164 & 14.18 & 1.10 & 0.10 & - & 5184 & .SAT3.. & 1 \\
\hline IC 184 & 015951.2 & -065028 & 7554 & 14.66 & 1.02 & 0.31 & 7 & 5400 & .SBR1* & 2 \\
\hline IC 1816 & 023151.3 & -364017 & 9634 & 13.83 & 1.16 & 0.07 & - & 5086 & .SBR2P? & 1 \\
\hline UGC 3223 & 045909.4 & +045831 & 16482 & 13.83 & 1.15 & 0.25 & 80 & 4723 & .SB.1.. & 1 \\
\hline NGC 2639 & 084338.0 & +501224 & 24506 & 12.59 & 1.26 & 0.22 & 140 & 3198 & RSAR1* & 1 \\
\hline IC 2510 & 094743.2 & -325015 & 28147 & 14 & 1.10 & 0.27 & 148 & 2700 & SBT2* & 2 \\
\hline NGC 3281 & 103152.2 & -345127 & 31090 & 12.60 & 1.52 & 0.30 & 140 & 3439 & .SAS2P* & 2 \\
\hline NGC 3660 & 112332.3 & -083929 & 34980 & 15 & 1.43 & 0.09 & 115 & 3678 & .SBR4.. & 2 \\
\hline NGC 4253 & 121826.4 & +294848 & 39525 & 14.00 & 0.98 & 0.06 & - & 3819 & PSBS1*. & 2 \\
\hline NGC 4507 & 123537.1 & -395431 & 41960 & 12.78 & 1.22 & 0.10 & - & 3499 & PSXT3.. & 2 \\
\hline NGC 4785 & 125326.9 & -484458 & 43791 & 13.21 & 1.29 & 0.31 & 81 & 3735 & PSBR3*. & 2 \\
\hline NGC 5347 & 135318.6 & +332932 & 49342 & 13.16 & 1.23 & 0.10 & 130 & 2312 & PSBT2.. & 2 \\
\hline NGC 5728 & 144224.1 & -171512 & 52521 & 12.37 & 1.49 & 0.24 & 0 & 2885 & .SXR1*. & 1 \\
\hline ESO $139-12$ & 173739.5 & -595629 & 60594 & 13.59 & 1.20 & 0.11 & 35 & 5200 & PSAT4P* & 2 \\
\hline NGC 6814 & 194240.7 & -101925 & 63545 & 11.85 & 1.48 & 0.03 & - & 1509 & .SXT4.. & 1 \\
\hline NGC 6860 & 200846.2 & -610556 & 64166 & 13.68 & 1.13 & 0.25 & 34 & 4462 & PSBR3.. & 1 \\
\hline NGC 6890 & 201817.9 & -444825 & 64446 & 13.05 & 1.19 & 0.10 & 152 & 2471 & .SAT3.. & 2 \\
\hline NGC 6951 & $2037 \quad 15.2$ & +660622 & 65086 & 11.91 & 1.59 & 0.08 & 170 & 1331 & $. \mathrm{SXT} 4 .$. & 2 \\
\hline \multicolumn{11}{|c|}{ Non-active galaxies } \\
\hline NGC 151 & 003402.8 & -094220 & 2035 & 12.23 & 1.57 & 0.34 & 75 & 3654 & .SBR4.. & \\
\hline IC 454 & 065106.6 & +125519 & 19725 & - & 1.24 & 0.28 & 140 & 3945 & .SB.2.. & \\
\hline NGC 2712 & 085931.2 & +445456 & 25248 & 12.38 & 1.46 & 0.26 & 178 & 1833 & .SBR3* & \\
\hline NGC 2811 & 091611.3 & -161847 & 26151 & 12.66 & 1.40 & 0.46 & 20 & 2514 & .SBT1.. & \\
\hline NGC 3571 & 111130.1 & -181719 & 34028 & 12.99 & 1.48 & 0.46 & 94 & 3571 & PSXT1*. & \\
\hline NGC 3835 & 114405.6 & +600713 & 36493 & 13.20 & 1.29 & 0.39 & 60 & 2452 & SX. $2^{* a} /$ & \\
\hline NGC 4162 & 121151.8 & +240724 & 38851 & 12.55 & 1.37 & 0.22 & 174 & 2542 & RSAT4.. & \\
\hline NGC 4290 & 122048.4 & +580532 & 39859 & 12.66 & 1.37 & 0.16 & 90 & 3035 & .SBT2* & \\
\hline NGC 4779 & 125350.8 & +094233 & 43837 & 12.91 & 1.33 & 0.07 & 70 & 2793 & .SBT4.. & \\
\hline NGC 6012 & 155413.5 & $\begin{array}{l}+143608 \\
\end{array}$ & 56334 & 12.96 & 1.32 & 0.14 & 168 & 1988 & RSBR2*. & \\
\hline NGC 6155 & 162608.4 & +482158 & 58115 & 13.20 & 1.13 & 0.16 & 145 & 2429 &. $\mathrm{SXT}_{.}{ }^{b}$ & \\
\hline
\end{tabular}

(1) Most common galaxy name;

(2) and (3) $\alpha$ and $\delta$ coordinates (equinox 2000.0);

(4) PGC number;

(5) $B$ magnitude;

(6) isophotal diameter (at $\left.B=25 \mathrm{mag} \operatorname{arcsec}^{-2}\right), D_{25}$, in units of $\log (0.1 \times a(\operatorname{arcmin}))$;

(7) axis ratio, $R_{25}$ in units of $\log (a / b)$;

(8) major axis position angle in degrees from $\mathrm{N}$ to $\mathrm{E}$ (when available);

(9) velocity in $\mathrm{km} \mathrm{s}^{-1}$;

(10) galaxy morphological type as given by the RC3, except for ${ }^{a}\left(. \mathrm{S} . .2^{*}\right.$ in the RC3) and ${ }^{b}(. \mathrm{S} ? \ldots$ in the RC3), for which the morphological type is from Márquez \& Moles (1996);

(11) Seyfert activity class for active galaxies.

The data in Cols. 5-9 were taken from the RC3.

As seen in Table 1, there are some galaxies with extended angular sizes close to 2 arcmin or even larger, which do not fit in a single frame. For two of them, namely NGC 4779 and NGC 6951, a mosaic of images was performed in order to have images for the entire galaxy disk.
The data reduction and calibration was performed, following the standard procedures with the $\mathrm{IRAF}^{3}$ software

${ }^{3}$ IRAF is the Image Analysis and Reduction Facility made available to the astronomical community by the National Optical Astronomy Observatories, which are operated by the 
Table 2. Journal of observations

\begin{tabular}{|c|c|c|c|c|c|c|}
\hline Name & Teles. & $\begin{array}{c}\text { Obs. } \\
\text { date }\end{array}$ & Filter & $\begin{array}{l}\text { Exp. } \\
\text { time } \\
(\mathrm{s})\end{array}$ & $\begin{array}{l}\text { Seeing } \\
\text { FWHM } \\
\text { (arcsec) }\end{array}$ & $\begin{array}{l}\mu_{2 \sigma} \\
(\mathrm{mag} / \\
\left.\operatorname{arcsec}^{2}\right)\end{array}$ \\
\hline UGC 1395 & CAHA & $09 / 96$ & $K^{\prime}$ & 5400 & 0.95 & 20.7 \\
\hline IC 184 & CAHA & $09 / 96$ & $K^{\prime}$ & 1800 & 0.95 & 19.8 \\
\hline IC 1816 & $\mathrm{LC}$ & $09 / 96$ & $\begin{array}{c}K^{\prime} \\
J\end{array}$ & $\begin{array}{r}3000 \\
800\end{array}$ & - & $\begin{array}{l}20.1 \\
21.0\end{array}$ \\
\hline UGC 3223 & $\mathrm{LC}$ & & $K^{\prime}$ & 2700 & 1.2 & 19.9 \\
\hline NGC $2639^{1}$ & TBL & $03 / 97$ & $\underset{K^{\prime}}{J}$ & $\begin{array}{l}1000 \\
3000\end{array}$ & - & $\begin{array}{l}20.5 \\
19.7\end{array}$ \\
\hline \multirow[t]{2}{*}{ IC $2510^{*}$} & $\mathrm{LC}$ & $05 / 96$ & $K^{\prime}$ & 3600 & 0.87 & 19.6 \\
\hline & & $04 / 97$ & $J$ & 1000 & 0.71 & 20.8 \\
\hline \multirow[t]{2}{*}{ NGC 3281} & $\mathrm{LC}$ & $05 / 96$ & $K^{\prime}$ & 3500 & 0.84 & 19.8 \\
\hline & $\mathrm{LC}$ & $\begin{array}{l}04 / 97 \\
04 / 97\end{array}$ & $\begin{array}{l}J \\
J\end{array}$ & $\begin{array}{l}1000 \\
1200\end{array}$ & $\begin{array}{l}0.89 \\
-\end{array}$ & $\begin{array}{l}21.1 \\
21.3\end{array}$ \\
\hline NGC 3660 & & $04 / 97$ & $K^{\prime}$ & 2000 & - & 20.2 \\
\hline NGC $4253^{3}$ & TBL & $03 / 97$ & $\begin{array}{c}J \\
K^{\prime}\end{array}$ & $\begin{array}{l}1000 \\
2800\end{array}$ & - & $\begin{array}{l}21.3 \\
19.3\end{array}$ \\
\hline NGC $4507^{*}$ & $\mathrm{LC}$ & $05 / 96$ & $J^{\prime}$ & $\begin{array}{l}1400 \\
3600\end{array}$ & $\begin{array}{l}0.80 \\
0.97\end{array}$ & $\begin{array}{l}21.0 \\
20.2\end{array}$ \\
\hline NGC $4785^{3}$ & $\mathrm{LC}$ & $04 / 97$ & $\begin{array}{c}\Lambda \\
J \\
K^{\prime}\end{array}$ & $\begin{array}{l}1000 \\
3000\end{array}$ & $\begin{array}{l}0.71 \\
0.71\end{array}$ & $\begin{array}{l}20.8 \\
20.2\end{array}$ \\
\hline NGC $5347^{*, 2}$ & TBL & $03 / 97$ & $\begin{array}{c}J \\
K^{\prime}\end{array}$ & $\begin{array}{l}1000 \\
3060\end{array}$ & $\begin{array}{l}1.4 \\
1.9\end{array}$ & $\begin{array}{l}21.3 \\
19.4\end{array}$ \\
\hline NGC 5728 & $\mathrm{LC}$ & $04 / 97$ & $\underset{K^{\prime}}{J}$ & $\begin{array}{l}1000 \\
2800\end{array}$ & $\begin{array}{l}0.88 \\
0.85\end{array}$ & $\begin{array}{l}20.9 \\
20.2\end{array}$ \\
\hline ESO 139-12 & $\mathrm{LC}$ & $05 / 96$ & $\underset{K^{\prime}}{J}$ & $\begin{array}{l}1000 \\
3400\end{array}$ & $\begin{array}{l}1.0 \\
0.97\end{array}$ & $\begin{array}{l}21.2 \\
19.5\end{array}$ \\
\hline NGC $6814^{3}$ & $\mathrm{LC}$ & $05 / 96$ & $\underset{K^{\prime}}{J}$ & $\begin{array}{l}1000 \\
3200\end{array}$ & $\begin{array}{l}1.2 \\
0.87\end{array}$ & $\begin{array}{l}20.9 \\
19.3\end{array}$ \\
\hline \multirow[t]{2}{*}{ NGC $6860^{3}$} & $\mathrm{LC}$ & $04 / 97$ & $\begin{array}{c}J \\
K^{\prime}\end{array}$ & $\begin{array}{l}1000 \\
2600\end{array}$ & $\begin{array}{l}0.87 \\
0.89\end{array}$ & $\begin{array}{l}20.9 \\
20.3\end{array}$ \\
\hline & $\mathrm{ESO}$ & $05 / 96$ & $K^{\prime}$ & 720 & 0.89 & 17.5 \\
\hline NGC 6890 & $\mathrm{LC}$ & $04 / 97$ & $\underset{K^{\prime}}{J}$ & 1000 & 0.76 & 20.7 \\
\hline NGC 6951 & NOT & $09 / 96$ & $\begin{array}{c}K^{\prime} \\
J \\
K^{\prime} \\
\end{array}$ & $\begin{array}{l}2600 \\
1760 \\
3520 \\
\end{array}$ & $\begin{array}{l}0.78 \\
1.3 \\
1.3 \\
\end{array}$ & $\begin{array}{l}20.1 \\
18.8 \\
19.5 \\
\end{array}$ \\
\hline NGC $151^{1}$ & $\mathrm{LC}$ & $09 / 96$ & $\underset{K^{\prime}}{J}$ & 800 & - & 21.1 \\
\hline IC 454 & TBL & $03 / 97$ & $\begin{array}{c}K^{\prime} \\
J \\
K^{\prime}\end{array}$ & $\begin{array}{l}1800 \\
1000 \\
2800\end{array}$ & $\begin{array}{l}- \\
1.2 \\
1.25\end{array}$ & $\begin{array}{l}19.9 \\
20.3\end{array}$ \\
\hline NGC 2712 & TBL & $03 / 97$ & $\begin{array}{c}\Lambda \\
J \\
K^{\prime}\end{array}$ & $\begin{array}{l}28000 \\
1000 \\
3200\end{array}$ & $\begin{array}{l}1.20 \\
1.2\end{array}$ & $\begin{array}{l}19.2 \\
20.7 \\
20.1\end{array}$ \\
\hline NGC $2811^{*}$ & $\mathrm{LC}$ & $05 / 96$ & $K^{\prime}$ & 3400 & 1.0 & 19.8 \\
\hline \multirow[t]{2}{*}{ NGC $3571^{1}$} & $\mathrm{LC}$ & $04 / 97$ & $\begin{array}{l}J \\
J \\
J\end{array}$ & $\begin{array}{l}1000 \\
1000\end{array}$ & $\begin{array}{l}1.19 \\
0.84\end{array}$ & $\begin{array}{l}20.7 \\
21.1\end{array}$ \\
\hline & & & $K^{\prime}$ & 3000 & 0.67 & 20.1 \\
\hline \multirow[t]{2}{*}{ NGC $3835^{*}$} & TBL & $03 / 97$ & $J$ & 1000 & - & 21.3 \\
\hline & & & $K^{\prime}$ & 3000 & - & 19.7 \\
\hline \multirow[t]{2}{*}{ NGC 4162} & TBL & $03 / 97$ & $J$ & 800 & 1.6 & 21.3 \\
\hline & & & $K^{\prime}$ & 3000 & 1.1 & 20.0 \\
\hline \multirow[t]{2}{*}{ NGC 4290} & TBL & $03 / 97$ & $J$ & 1200 & 1.1 & 21.0 \\
\hline & & & $K^{\prime}$ & 3000 & 1.1 & 19.5 \\
\hline \multirow[t]{2}{*}{ NGC 4779} & TBL & $03 / 97$ & $\underset{K^{\prime}}{J}$ & $4 \times 1000$ & 1.1 & $\begin{array}{l}20.9 \\
19.3\end{array}$ \\
\hline & & & $K^{\prime}$ & $4 \times 1000$ & $\begin{array}{l}1.1 \\
1.2\end{array}$ & 19.3 \\
\hline NGC 6012 & TBL & $03 / 97$ & $\underset{K^{\prime}}{J}$ & $\begin{array}{l}1000 \\
3600\end{array}$ & $\begin{array}{l}1.2 \\
1.35\end{array}$ & $\begin{array}{l}21.0 \\
19.7\end{array}$ \\
\hline \multirow[t]{2}{*}{ NGC 6155} & TBL & $03 / 97$ & $J$ & 600 & 1.15 & 21.0 \\
\hline & & & $K^{\prime}$ & 3200 & 1.65 & 19.5 \\
\hline
\end{tabular}

CAHA: $3.5 \mathrm{~m}$ telescope at Calar Alto $\left(0.32^{\prime \prime} / \mathrm{pixel}\right)$; LC: $2.5 \mathrm{~m}$ telescope at Las Campanas $\left(0.348^{\prime \prime} / \mathrm{pixel}\right)$; TBL: $2 \mathrm{~m}$ Bernard Lyot telescope at Pic du Midi $\left(0.5^{\prime \prime} / \mathrm{pixel}\right)$; ESO: $2.2 \mathrm{~m}$ telescope at La Silla $\left(0.278^{\prime \prime} / \mathrm{pixel}\right)$; NOT: Nordic Optical Telescope at La Palma. $\left(0.51^{\prime \prime} / \mathrm{pixel}\right)$.

* Galaxies with non-flat remaining background.

1,2,3 Galaxies with NICMOS HST archive images, (1) published by Böker et al. (1999); (2) published by Regan \& Mulchaey (1999); (3) unpublished.

and SQIID package. For the objects, exposures of typically about 2 minutes (dithered in short exposures of about

Association of Universities for Research in Astronomy (AURA), Inc., under contract with the U.S. National Science Foundation.
$20 \mathrm{~s}$ in $J$ and $50 \mathrm{~s}$ in $K^{\prime}$ offset by about $2-3 \operatorname{arcsec}$ ) were taken on the source and on the sky, until the total integration time was achieved (see Table 2). A median dark frame was calculated for every night with the same exposure as that of the objects by using the corresponding dark frames obtained before and after the observations. 
Flat-field frames were obtained for each filter by subtracting the median dark frame from the sky frames, normalizing the resulting frames and calculating the median. We then subtracted the dark frame from the object frame, divided by the normalized flat-field and subtracted the corresponding sky frame. Sometimes, another constant had to be subtracted in order to attain the zero background level. In order to align the resulting frames to better than a pixel fraction we used the non-saturated stars in these frames or, when there were no stars, we used the centre of the galaxy. For the flux calibration a number of standard stars were observed and mean extinction coefficients were applied $\left(k_{J}\right.$ and $k_{K^{\prime}}$ in magnitudes airmass ${ }^{-1}$ are respectively 0.12 and 0.08 for Las Campanas and La Silla, 0.12 and 0.10 for the TBL, 0.057 and 0.042 for the NOT and $k_{K^{\prime}}=0.1$ for Calar Alto). The error level on the standard stars amounts to $10 \%$. In Col. 7 of Table 2 we give the isophotal magnitude/ $\operatorname{arcsec}^{2}$ corresponding to $2 \sigma$ of the background.

We have retrieved HST calibrated infrared images obtained with NICMOS (filter F160W) for the eight galaxies for which such data was available (see Col. 1 in Table 2).

\section{Methods of analysis}

\subsection{Morphological features}

$K^{\prime}$ images of the galaxies in grey scale with isocontours overlaid are shown in Figs. 1a-28a. All figures are only available in electronic form.

In order to detect the presence of features showing a departure from radial symmetry such as bars and spiral arms, we have used a masking technique. We filtered the original images with a box of $2-3$ times the FWHM of the seeing (median filter) and divided the observed image by the filtered one. The resulting images (hereafter called the sharp-divided images to differenciate them from those obtained by subtraction, i.e. the so-called unsharp masking technique) are shown in Figs. 1b-28b. This technique is very well suited to trace asymmetries in the light distribution, such as bars, spiral arms, dust lanes, rings; it allows the subtraction of the diffuse background in a very convenient way to look for subtle, small-scale variations and discuss the possible presence of both dust extinguished and more luminous regions (Sofue et al. 1994; Márquez \& Moles 1996; Márquez et al. 1996; Erwin \& Sparke 1999; Laine et al. 1999).

The presence of bars can be determined quantitatively by studying the behaviour of isophotal position angles (PAs) and ellipticities $(\epsilon \mathrm{S})$ : a bar is characterized by a local maximum in the $\epsilon$ corresponding to a constant PA (Wozniak et al. 1995; Friedli et al. 1996; Jungwiert et al. 1997). Therefore, we have fit ellipses to the isophotes (Jedrzejewski 1987) with the IRAF ellipse task in stsdas.analysis.isophote, allowing the center, PA and $\epsilon$ to vary from one isophote to the next. Foreground stars have been masked out. The resulting parameters allow the reconstruction of a model galaxy. The difference between the original image and the model (hereafter called the difference images) are particularly useful to trace all the features that cannot be described with ellipses, as spiral arms and boxy or peanut-like components. They are given in Figs. 1c-28c and discussed separately for each galaxy. When the PA suddenly changes from one isophote to the next, the model cannot work for the regions left inside (see Figs. 1c, 8c, 9c, 11c, 12c, 15c, 18c, 19c, 25c and 26c).

\subsection{Surface photometry and photometric decomposition}

The major axis PA (counted anticlockwise from north to east as usual) and $\epsilon$ from the ellipse fitting are drawn as a function of the ellipse semi-major axis (therefore, in radius) in Figs. 1e-28e.

In Figs. 1f-28f the surface brightness profiles (isophotal magnitude fit to the ellipse versus ellipse semi-major axis) are given for $K^{\prime}$ and $J$. We have used the 1-D resulting profiles to obtain the bulge and disk contributions. We have fit an exponential to the outermost region (the disk), subtracted it to the observed profile and fit a $r^{1 / 4}$ law (the bulge) to the residual; this calculation was continued until convergence was achieved (see Márquez \& Moles 1996, 1999). The resulting bulge and disk parameters are given in Table 3 . The surface brightness profiles in $J$ and $K^{\prime}$ are plotted in Figs. 1f-28f with the bulge, disk and bulge+disk fits superimposed. The corresponding residuals are shown in Figs. 1g-28g. Since residual background subtraction is the biggest source of error in determining the profiles (see for instance de Jong 1996), we caution the reader that in the cases where the galaxy occupies most of the frame and the residual background level could only be determined in very small regions, the error in the profiles can reach $15-20 \%$. Otherwise, residuals are in general smaller than $20 \%$, except in the bar and spiral arm regions.

\subsection{Color images and color gradients}

For galaxies for which a $J$ band image is also available, we display in Figs. 1d-28d the ratio of the $J$ to $K^{\prime}$ images (after background subtraction), median filtered by $3 \times 3$ pixels in order to enhance the $\mathrm{S} / \mathrm{N}$ of the outer regions. Color images will allow a more complete description of the previously detected morphological features in terms of the contribution of dust and/or star forming events. We note that in the cases where the background is not flat, somewhat structured color images are obtained (galaxies with non-flat remaining backgrounds are indicated with an asterisk in Col. 1 of Table 2).

Magnitudes integrated in circular apertures and $J-K^{\prime}$ color gradients were obtained from the curves of growth as 
Table 3. Bulge+disk decomposition parameters and magnitudes

\begin{tabular}{|c|c|c|c|c|c|c|c|c|c|c|c|c|c|c|c|c|c|c|}
\hline Galaxy & $\begin{array}{r}\mu_{\mathrm{d}} \\
J\end{array}$ & $r_{\mathrm{d}}$ & $m_{\mathrm{d}}$ & $\mu_{\mathrm{b}}$ & $r_{\mathrm{b}}$ & $m_{\mathrm{b}}$ & $m_{\mathrm{T}}$ & $\begin{array}{l}\mu_{\mathrm{d}} \\
K^{\prime}\end{array}$ & $r_{\mathrm{d}}$ & $m_{\mathrm{d}}$ & $\mu_{\mathrm{b}}$ & $r_{\mathrm{b}}$ & $m_{\mathrm{b}}$ & $m_{\mathrm{T}}$ & $m_{J}^{\mathrm{ap}}$ & $r_{J}^{\mathrm{ap}}$ & $m_{K^{\prime}}^{\mathrm{ap}}$ & $r_{K^{\prime}}^{\mathrm{ap}}$ \\
\hline (1) & (2) & (3) & (4) & (5) & (6) & $(7)$ & $(8)$ & (9) & (10) & (11) & (12) & (13) & (14) & (15) & (16) & (17) & (18) & (19) \\
\hline UGC 1395 & - & - & - & - & - & - & - & 19.8 & 21 & 10.5 & 16.3 & 1.17 & 12.6 & 10.4 & - & - & 10.82 & 35 \\
\hline IC 184 & - & - & - & - & - & - & - & 18.2 & 14 & 9.9 & 17.7 & 4.9 & 10.9 & 9.5 & - & - & 9.98 & 35 \\
\hline IC 1816 & 18.8 & 11 & 11.0 & 11.2 & 0.2 & 10.9 & 10.2 & 18.1 & 13 & 9.8 & 12.6 & 0.54 & 10.56 & 9.38 & 10.65 & 45 & 9.52 & 45 \\
\hline UGC 3223 & & & & & & & & 18.1 & 20 & 8.9 & 15.9 & 2.1 & 10.9 & 8.7 & & & 9.28 & 45 \\
\hline NGC 2639 & 17.6 & 17 & 8.7 & 18.2 & 9.6 & 9.9 & 8.4 & 16.7 & 18 & 7.8 & 17.5 & 14 & 8.3 & 7.3 & 9.08 & 50 & 7.83 & 50 \\
\hline IC 2510 & 19 & 19.2 & 10.1 & 18.0 & 2.9 & 12.3 & 10. & 17.9 & 16 & 9.1 & 10.6 & 0.3 & 10.2 & 8.8 & 10.82 & 42 & 9.56 & 42 \\
\hline NGC 3281 & 19.6 & 92 & 7.03 & 18.8 & 15 & 9.48 & 6.9 & 19.1 & 109 & 6.2 & 17.8 & 17 & 8.3 & 6.1 & 9.42 & 35 & 8.43 & 35 \\
\hline NGC 3660 & 21.2 & 44 & 10.3 & 21.4 & 57 & 9.3 & 8.9 & 19.1 & 18 & 10 & 19.8 & 19 & 10 & 9.4 & 10.63 & 35 & 9.89 & 20 \\
\hline NGC 4253 & 18.4 & 10 & 10.7 & 17.3 & 2.7 & 11.7 & 10.3 & 17.0 & 10 & 9.4 & 6.4 & 0.1 & 7.3 & 7.2 & 10.63 & 50 & 9.21 & 50 \\
\hline NGC 4507 & 20.1 & 31 & 9.9 & 17.9 & 7.5 & 10.1 & 9.3 & 18.9 & 32 & 8.7 & 16.0 & 4.8 & 9.2 & 8.2 & 9.97 & 44 & 8.75 & 44 \\
\hline NGC 4785 & 17.1 & 22 & 7.7 & 12.3 & 0.7 & 9.7 & 7.6 & 17.5 & 25 & 7.8 & 15.0 & 2.1 & 9.9 & 7.6 & 9.73 & 28 & 8.77 & 28 \\
\hline NGC 5347 & 19.8 & 23 & 10.3 & 19.1 & 7.9 & 11.2 & 9.9 & 18.2 & 19 & 9.1 & 15.5 & 2.2 & 10.4 & 8.8 & 10.30 & 48 & 9.00 & 48 \\
\hline NGC 5728 & 20.2 & 103 & 7.4 & 17.0 & 6.1 & 9.6 & 7.3 & 18.1 & 22 & 8.8 & 15.3 & 3.6 & 9.1 & 8.2 & 9.58 & 38 & 8.68 & 38 \\
\hline ESO 139 & 20.0 & 25 & 10.3 & 15.6 & 1.3 & 11.6 & 10. & 19.1 & 26 & 9.3 & 15.2 & 1.7 & 10.6 & 9.0 & 10.54 & 45 & 9.69 & 45 \\
\hline NGC 6814 & 19.4 & 62 & 7.7 & 18.5 & 9.2 & 10.3 & 7.6 & 18.0 & 40 & 7.3 & 16.6 & 5.5 & 9.5 & 7.1 & 9.30 & 47 & 8.04 & 47 \\
\hline NGC 6860 & 18.2 & 11 & 10.3 & 13.2 & 0.4 & 11.5 & 10. & 18.1 & 15 & 9.6 & 16.6 & 3.9 & 10.3 & 9.1 & 10.56 & 38 & 9.49 & 38 \\
\hline NGC 6890 & 18.4 & 16 & 9.7 & 17.8 & 3.5 & 11.7 & 9.6 & 17.2 & 15 & 8.6 & 15.5 & 1.8 & 10.8 & 8.5 & 10.35 & 29 & 8.97 & 38 \\
\hline NGC 6951 & 20.4 & 69 & 8.5 & 19.4 & 22 & 9.3 & 8.1 & 18.6 & 55 & 7.2 & 17.4 & 16 & 8.0 & 6.8 & 8.6 & 112 & 7.20 & 112 \\
\hline NGC 151 & 23.8 & 191 & 9.7 & 19.6 & 25 & 9.3 & 8.7 & 19.3 & 11 & 11.4 & 19.2 & 15 & 10.0 & 9.7 & 9.18 & 42 & 8.68 & 45 \\
\hline IC 454 & 19.4 & 53 & 8.1 & 18.2 & 5.8 & 11.0 & 8.0 & 18.1 & 74 & 6.1 & 16.6 & 5.5 & 9.5 & 6.0 & 10.02 & 45 & 8.77 & 45 \\
\hline NGC 2712 & 19.0 & 24 & 9.5 & 10.2 & 0.3 & 9.4 & 8.7 & 18.3 & 26 & 8.5 & 13.5 & 1.5 & 9.3 & 8.1 & 9.69 & 55 & 8.59 & 55 \\
\hline NGC 2811 & 19.3 & 47 & 8.2 & 17.4 & 9.8 & 9.0 & 7.8 & 17.4 & 26 & 7.6 & 14.6 & 3.6 & 8.4 & 7.2 & 9.07 & 38 & 7.92 & 38 \\
\hline NGC 3571 & 20.1 & 68 & 8.3 & 18.4 & 9.0 & 10.3 & 8.1 & 19.3 & 50 & 8.1 & 17.3 & 7.9 & 9.4 & 7.8 & 10.09 & 38 & 9.13 & 38 \\
\hline NGC 3835 & 18.8 & 25 & 9.1 & 16.9 & 2.7 & 11.4 & 8.9 & 17.8 & 23 & 8.3 & 18.4 & 12 & 9.7 & 8.0 & 10.22 & 55 & 8.86 & 55 \\
\hline NGC 4162 & 18.7 & 17 & 9.8 & 17.3 & 2.7 & 11.8 & 9.6 & 17.5 & 18 & 8.6 & 13.7 & 0.9 & 10.5 & 8.4 & 9.78 & 55 & 8.64 & 55 \\
\hline NGC 4290 & 18.6 & 20 & 9.4 & 13.7 & 0.7 & 11.2 & 9.2 & 17.6 & 20 & 8.3 & 12.6 & 0.7 & 9.9 & 8.1 & 9.74 & 60 & 8.46 & 60 \\
\hline NGC 4779 & 20.4 & 37 & 9.8 & 16.0 & 1.8 & 11.4 & 9.6 & 19.4 & 37 & 8.9 & 14.8 & 1.7 & 10.2 & 8.6 & 10.06 & 65 & 8.83 & 65 \\
\hline NGC 6012 & 19.1 & 30 & 9.1 & 16.6 & 0.8 & 13.8 & 9.0 & 18.0 & 30 & 7.9 & 11.8 & 0.2 & 11.6 & 7.8 & 10.00 & 48 & 8.90 & 48 \\
\hline NGC 6155 & 19.2 & 19 & 10.2 & 20.3 & 11.8 & 11.5 & 9.9 & 18.5 & 18 & 9.5 & 20.7 & 52 & 9.2 & 8.6 & 10.29 & 55 & 9.19 & 55 \\
\hline
\end{tabular}

(1) Galaxy name.

(2) Equivalent surface brightness for the disk component in $J$ band

(3) Equivalent radius for the disk component in $J$ band.

(4) Computed total magnitude for the disk in $J$ band.

(5) Equivalent surface brightness for the bulge component in $J$ band.

(6) Equivalent radius for the bulge component in $J$ band.

(7) Computed total magnitude for the bulge in $J$ band.

(8) Computed total bulge + disk $J$ magnitude.

(9)-(15) The same as (2)-(8) for the $K^{\prime}$ band.

(16) $J$ magnitude measured from simulated aperture photometry.

(17) Radius of the circular aperture used to measure the $J$ magnitude.

(18) $K^{\prime}$ magnitude measured from simulated aperture photometry.

(19) Radius of the circular aperture used to measure the $K^{\prime}$ magnitude.

Surface brightnesses are given in magnitudes $\operatorname{arcsec}^{-2}$ and radii in arcsec

in Márquez \& Moles (1996). The apertures and the corresponding magnitudes are given in Table 3. Color gradients are shown in Figs. 1h-28h for galaxies for which we have data in the two bands. The background subtraction was done as described above and the same caveat applies, in the sense that some color gradient could be artificially produced for the galaxies with non-flat backgrounds.

In Table 4 we give the magnitudes that we measure for 9 galaxies, together with $J, K^{\prime}$ and/or $K$ magnitudes found in the literature. A straightforward comparison between our $J$ values and those reported in previous works (for the same apertures) gives: $J_{\mathrm{us}}-J_{\text {other }}=-0.42 \pm 0.25$. Only 4 galaxies in our sample have been observed in $K^{\prime}$ by Mulchaey et al. (1997); we find: $K_{\text {us }}^{\prime}-K_{\text {Mulchaey }}^{\prime}=$
$0.015 \pm 0.63$. If we exclude NGC 6890 (see Sect. 5.17) we obtain: $K_{\text {us }}^{\prime}-K_{\text {Mulchaey }}^{\prime}=-0.30 \pm 0.36$. For 10 galaxies and a total of 12 measurements, we find: $K_{\text {us }}^{\prime}-K_{\text {other }}=$ $-0.42 \pm 0.32$. This value is comparable to the difference between $K_{\text {Mulchaey }}^{\prime}$ and $K_{\text {other }}(-0.55 \pm 0.49)$. These differences in the zero points are not critical for our purposes and will not be discussed further. In any case, we notice that the differences in the colour indexes $J-K^{\prime}$ are within the errors.

In Table 5 we give the parameters we determine for primary and secondary bars ${ }^{4}$ in the sample galaxies. We

\footnotetext{
${ }^{4}$ We note that all the elongated central structures we call secondary bars could be either bars or elongated rings or disks; kinematical data will help to discriminate among them.
} 
Table 4. Total magnitudes for the 9 galaxies with also $K^{\prime}$ and/or $J$ magnitudes from the litterature

\begin{tabular}{lrrrr}
\hline Galaxy & $K_{\text {us }}^{\prime}$ & $K_{\text {others }}^{\prime}($ ref $)$ & $J_{\text {us }}$ & $J_{\text {others }}($ ref $)$ \\
\hline UGC 1395 & 10.82 & $10.3(2)$ & & \\
NGC 2639 & 7.83 & $8.44(5)$ & 9.08 & $9.40(5)$ \\
NGC 3281 & 8.43 & $9.12(1)$ & 9.42 & $10.28(1)$ \\
NGC 4253 & 9.21 & $9.9(2), 9.56(5), 9.65(6)$ & 10.63 & $11.04(5), 11.01(6)$ \\
NGC 4507 & 8.75 & $9.28(1), 8.9(4)$ & 9.97 & $10.27(1)$ \\
NGC 5347 & 9.00 & $9.7(2), 9.8(4)$ & & $10.25(1)$ \\
NGC 5728 & 8.68 & $9.21(1)$ & 9.58 & $9.72(1)$ \\
NGC 6814 & 8.04 & $8.65(1), 8.0(4)$ & 9.30 & $10.31(1)$ \\
NGC 6890 & 8.97 & $9.26(1), 8.0(4)$ & 10.35 & \\
NGC 6012 & 9.02 & $9.19(3)$ & & \\
& \multicolumn{5}{c}{}
\end{tabular}

(1) $K$ magnitudes from Glass \& Moorwood (1985) (2) McLeod \& Rieke (1995) (3) de Jong (1996) (4) Alonso-Herrero et al. (1998) (5) Hunt et al. (1999) (6) $K^{\prime}$ magnitudes from Mulchaey et al. (1997).

specify which of the methods above have been used to detect the presence of secondary bars (or primary bars when detected for the first time), and if evidence is found in available HST images. We note that, excepting the secondary bar of IC 2510, only seen in the sharp-divided image (just marginally in the PA- $\epsilon$ plot), the secondary bars are obtained in at least two methods.

\section{Description of individual active galaxies}

\section{1. $U G C 1395$}

The $K^{\prime}$ image in Fig. 1a shows an extended bulge from which two spiral arms originate. A small elongation is seen in the center, that could be due to the presence of a small bar (see below). Although this galaxy is classified as $\mathrm{S}(\mathrm{rs}) \mathrm{b}$ in the RC3, McLeod \& Rieke (1995) already reported the presence of a bar with a 16 arcsec radius, $\mathrm{PA}=145^{\circ}$ and $\epsilon=0.5$ from their $K$ image. It doesn't show up in our sharp-divided image in Fig. 1b, but is clearly detected in the plot of $\epsilon$ and PA with radius (Fig. 1e) which is in agreement with Peletier et al. (1999). The parameters we deduce for the bar from that plot are very similar to those reported by McLeod \& Rieke (1995).

The sharp-divided image reveals the presence of a small bar, extending along $\mathrm{PA}=139^{\circ}$, up to $7 \operatorname{arcsec}$ from the center. It is not detected in the PA and $\epsilon$ plot as it is too weak to be apparent. Other evidence for this nuclear bar is the curved dust pattern that surrounds the very central region in the broad band HST image (filter F606W) by Malkan et al. (1998).

The difference image in Fig. 1c shows that the overall fit is good except in the region where the arm contribution is important. The surface brightness profile (in agreement with that of McLeod \& Rieke) is well fit by a bulge+disk model, except in the zone where the arm contribution is important (Figs. 1f and 1g).

\subsection{IC 184}

This galaxy shows rotation of the PA and twisting of the isophotes in the central region. Evidence for the existence of a bar inside the primary bar is presented in Figs. 2b and $2 \mathrm{e}$, where $\epsilon$ shows two maxima for a rather constant PA. The inner bar is also evident in the broad band HST image by Malkan et al. (1998). The region connecting the two bars is visible in Fig. $2 \mathrm{~b}$ as a thin curved elongation starting at the end of the inner (thicker) bar.

The difference image in Fig. 2c shows the two nested bars as well as the region where the spiral arms begin; the spiral arm to the north is much brighter than its southern counterpart. Due to the bars and arms, the residuals are high except in the very outer zones (Fig. $2 \mathrm{~g}$ ), and the bulge + disk fit is not very good (Fig. 2f).

\subsection{IC 1816}

A big bar (Fig. 3e) and three spiral arms are detected, among which the north west arm is the brightest and most detached (Fig. 3a). At large scales, the image has a somewhat triangular shape. A small galaxy is seen 35 arcsec to the south along $\mathrm{PA}=168^{\circ}$, but no redshift is available for it.

The sharp-divided image (Fig. 3b) clearly reveals the bar, the ring, and the spiral arms. Note that the very central zone appears somewhat elongated.

The bar and arms are also clearly seen in the difference image (Fig. 3c), and except for the bar region the residuals are quite small (Fig. $3 \mathrm{~g}$ ). The bulge + disk model fits the data very well except for the bar region (Fig. 3f). 
Table 5. Parameters of the bar(s)

\begin{tabular}{|c|c|c|c|c|c|c|c|c|}
\hline Galaxy & $\begin{array}{r}\text { Primary } \\
\text { bar PA }\end{array}$ & $\begin{array}{l}\text { Primary } \\
\text { bar } \\
\epsilon\end{array}$ & $\begin{array}{c}\text { Primary } \\
\text { bar size } \\
(\operatorname{arcsec})\end{array}$ & Detection* & $\begin{array}{r}\text { Secondary } \\
\text { bar PA }\end{array}$ & $\begin{array}{l}\text { Secondary } \\
\text { bar } \\
\epsilon\end{array}$ & $\begin{array}{l}\text { Secondary } \\
\text { bar size } \\
(\operatorname{arcsec})\end{array}$ & Detection* \\
\hline UGC 1395 & 145 & 0.56 & 16 & $\mathrm{f}, \mathrm{o}$ & 139 & 0.32 & 7 & $\mathrm{~s}, \mathrm{~h}$ \\
\hline IC 184 & 170 & 0.50 & 9 & $\mathrm{i}, \mathrm{f}, \mathrm{d}$ & 30 & 0.40 & 4 & $\mathrm{f}, \mathrm{h}$ \\
\hline IC 1816 & 110 & 0.50 & 10 & $\mathrm{i}, \mathrm{s}, \mathrm{f}, \mathrm{d}$ & $0:$ & $0.20:$ & 1: & $\mathrm{c}, \mathrm{h}$ \\
\hline UGC 3223 & 75 & 0.71 & 21 & $\mathrm{i}, \mathrm{s}, \mathrm{f}, \mathrm{d}$ & $75:$ & $0.45:$ & $5:$ & $\mathrm{f}, \mathrm{h}$ \\
\hline NGC 2639 & 137 & 0.40 & 8 & $\mathrm{f}, \mathrm{s}$ & 117 & 0.15 & 2 & $\mathrm{~s}, \mathrm{~h}$ \\
\hline IC 2510 & 145 & 0.61 & 15 & $\mathrm{i}, \mathrm{s}, \mathrm{f}$ & 143: & $0.52:$ & 7: & $\mathrm{s}$ \\
\hline NGC 3281 & 133 & 0.40 & 5 & $\mathrm{i}, \mathrm{s}, \mathrm{o}$ & & & & \\
\hline NGC 3660 & 110 & 0.70 & 23 & $\mathrm{i}, \mathrm{s}, \mathrm{f}, \mathrm{d}$ & & & & \\
\hline NGC 4253 & 105 & 0.50 & 11 & $\mathrm{i}, \mathrm{s}, \mathrm{f}, \mathrm{d}$ & 5 & 0.10 & 2 & $\mathrm{~s}, \mathrm{~d}, \mathrm{~h}$ \\
\hline NGC 4507 & 52 & 0.35 & 9 & $\mathrm{~s}, \mathrm{~d}, \mathrm{f}, \mathrm{o}$ & & & & \\
\hline NGC 4785 & 65 & 0.50 & 10 & $\mathrm{i}, \mathrm{f}$ & 82 & 0.35 & 5 & $\mathrm{f}, \mathrm{d}, \mathrm{c}, \mathrm{h}$ \\
\hline NGC 5347 & 105 & 0.60 & 34 & $\mathrm{i}, \mathrm{d}, \mathrm{f}$ & & & & \\
\hline NGC 5728 & 30 & 0.60 & 44 & $\mathrm{i}, \mathrm{f}, \mathrm{o}$ & 90 & 0.45 & 4 & $\mathrm{i}, \mathrm{s}, \mathrm{f}, \mathrm{d}$ \\
\hline \multicolumn{9}{|l|}{ ESO 139-12 } \\
\hline NGC 6814 & 25 & 0.30 & 12 & $\mathrm{i}, \mathrm{s}, \mathrm{f}, \mathrm{o}$ & & & & \\
\hline NGC 6860 & 10 & 0.55 & 10 & $\mathrm{i}, \mathrm{s}, \mathrm{f}$ & 90 & 0.10 & 4 & $\mathrm{f}, \mathrm{d}, \mathrm{s}, \mathrm{c}, \mathrm{h}$ \\
\hline NGC 6890 & 15 & 0.30 & 6 & $\mathrm{~s}, \mathrm{f}, \mathrm{o}$ & & & & \\
\hline NGC 6951 & 84 & 0.52 & 45 & $\mathrm{i}, \mathrm{s}, \mathrm{f}, \mathrm{d}, \mathrm{o}$ & & & & \\
\hline NGC 151 & 155 & 0.45 & 18 & $\mathrm{i}, \mathrm{s}, \mathrm{f}, \mathrm{d}$ & 80 & 0.30 & 5 & $\mathrm{~s}, \mathrm{f}, \mathrm{d}, \mathrm{h}, \mathrm{c}$ \\
\hline IC 454 & 128 & 0.76 & 18 & $\mathrm{i}, \mathrm{s}, \mathrm{f}, \mathrm{d}$ & 133 & 0.40 & 3 & $\mathrm{~s}, \mathrm{f}, \mathrm{c}$ \\
\hline NGC 2712 & 25 & 0.60 & 20 & $\mathrm{i}, \mathrm{d}, \mathrm{f}$ & 5 & 0.25 & 5 & $\mathrm{~s}, \mathrm{f}, \mathrm{d}, \mathrm{c}$ \\
\hline NGC 2811 & 30 & 0.50 & 20 & $\mathrm{~s}, \mathrm{f}$ & 20 & 0.30 & 5 & $\mathrm{~s}, \mathrm{f}, \mathrm{d}$ \\
\hline NGC 3571 & 90: & $0.25:$ & $2:$ & $\mathrm{s}, \mathrm{c}, \mathrm{h}, \mathrm{f}$ & & & & \\
\hline NGC 3835 & 55 & 0.40 & 5 & $\mathrm{~s}, \mathrm{f}, \mathrm{c}$ & & & & \\
\hline NGC 4162 & 145: & $0.30:$ & 12: & $\mathrm{f}, \mathrm{s}$ & & & & \\
\hline NGC 4290 & 35 & 0.65 & 26 & $\mathrm{i}, \mathrm{s}, \mathrm{f}, \mathrm{d}$ & $55:$ & $0.35:$ & $6:$ & $\mathrm{s}, \mathrm{f}, \mathrm{d}$ \\
\hline NGC 4779 & 8 & 0.65 & 30 & $\mathrm{f}, \mathrm{d}$ & & & & \\
\hline NGC 6012 & 155 & 0.65 & 16 & $\mathrm{i}, \mathrm{s}, \mathrm{f}, \mathrm{d}$ & & & & \\
\hline NGC 6155 & 160: & $0.35:$ & 15: & $\mathrm{i}, \mathrm{f}$ & 120 & 0.40 & 6 & $\mathrm{~s}, \mathrm{f}, \mathrm{d}$ \\
\hline
\end{tabular}

* We refer to the image in which the bar is detected as following: i: direct image; s: sharp-dividing; f: ellipse fitting parameters; d: difference image (original - model); c: color image; h: HST image; o: others (see text).

Notes:

All the elongated central structures we call secondary bars could be either bars or elongated rings or disks; kinematical data will help to discriminate among them.

The quantities followed by a column are those for which we are not certain that such an elongation is detected.

The $J / K^{\prime}$ image (Fig. 3d) has a rather smooth aspect except at the very center where a small, elongated structure is seen, just in the direction traced by the offset dust lane in the broad band HST image by Malkan et al. (1998). It could be the signature of a nuclear bar within a 1 arcsec radius (see also Fig. 3e).

\section{4. $U G C 3223$}

This is a classical barred SBa galaxy, with a very large bar (Figs. 4a and 4e). The sharp-divided image (Fig. 4b) clearly reveals the bar as well as the beginning of two spiral arms originating at both extremities of the bar.

The bulge + disk model fits well the observed profile except in the bar region (Fig. 4f), and except for this 
(large) region the residuals are very small (Fig. 4g). The spiral arms appear most clearly in the difference image (Fig. 4c).

We find evidence for the presence of an inner bar in Fig. 4e (see Table 5). In fact, an inner bar feature is evident in the HST broad band image by Malkan et al. (1998), with a curved dust lane structure associated with it.

\subsection{NGC $2639(U G C 4544)$}

This galaxy appears to be very regular at first sight (Fig. 5a). However, the sharp-divided image (Fig. 5b) reveals the presence of a fat primary bar, at variance with its $\mathrm{RSa}(\mathrm{r})$ classification. We note that Fig. 5e shows a roughly constant PA value for the whole galaxy, precluding a definitive quantification of the primary bar extension. In any case, NGC 2639 has to be classified as barred (or lensed) on the basis of the IR images.

The difference image reveals the existence of two spiral arms, one to the north west and the other to the south east, which splits into two arms (Fig. 5c). The surface brightness profile (in agreement with that by Moriondo et al. 1998) decomposition, specially in the $K^{\prime}$ band (Fig. 5f), implies very small residuals except in the lense and the spiral arm region (Fig. $5 \mathrm{~g}$ ).

A red arc-like feature going from the SE spiral arm northwards to the NW arm is visible in Fig. 5d, corresponding to the presence of dust lanes which clearly appear in the broad band HST image by Malkan et al. (1998). This would indicate the presence of a small central bar. An elongated ring-like structure from 9 to 18 arcsec in radius is seen in the Pa $\alpha$ HST image by Böker et al. (1999), i.e., a ring just exterior to the bar. This small bar is further confirmed by the sharp-dividing method and the ellipse fitting applied to the HST H image (see Figs. 5e and 29).

\section{6. $I C 2510$}

The $K^{\prime}$ band image shows a bar, spiral arms and a pseudoring (Fig. 6a). The spiral arm to the south is clearly visible on the sharp-divided image (Fig.6b), as well as a secondary bar.

The difference image (Fig. 6c) shows very distinctly the spiral. This is the only case for which we detect a secondary bar only from one method. Moreover, primary and secondary bars have almost the same PA. We therefore quote the secondary bar as doubtful in Table 5 .

The bulge + disk decomposition gives good results except in the bar region, specially in $K^{\prime}$ (Figs. 6f and 6g). The $J / K^{\prime}$ image remains quite constant (Figs. $6 \mathrm{~d}$ and $6 \mathrm{~h}$ ).

\section{7. $N G C 3281$}

The $K^{\prime}$ image (Fig. 7a) shows an elongated boxy bulge with possible spiral arms coming out of it. This galaxy is classified as non-barred in the RC3. However, the sharpdivided image (Fig. 7b) clearly shows the existence of a weak bar. The possible existence of a bar was already suggested by Xanthopoulos (1996), on the basis of an I band image. We also detect weak spiral arms in the sharpdivided image. Since these structures are weak, the PA is roughly constant throughout the galaxy (Fig. 7e); however, they do induce a small bump in the surface brightness profiles (Fig. 7f).

The $J / K^{\prime}$ image (Fig. $7 \mathrm{~d}$ ) shows that the $\left(J-K^{\prime}\right)$ color index is fainter in the nucleus, in the small spiral structure around the nucleus, and in the region of the bar.

\subsection{NGC 3660 (Mrk 1291)}

A strong bar is seen in the $K^{\prime}$ image of this galaxy (Fig. 8a) but the ring is barely visible. On the other hand, both the bar and ring appear clearly on the sharp-divided image (Fig. 8b).

The edges of the bar can also be seen in the difference image (Fig.8c); the bar parameters are determined from Fig. 8e. From an $R$ image, Chapelon et al. (1999) give $\mathrm{PA}=116^{\circ}$ out to 16 arcsec for the bar. This agrees with Friedli et al. (1996) who show that bars are generally longer in $K$ than in $R$.

The $J / K^{\prime}$ color image (Fig. 8d) shows a smooth structure, increasingly red towards the center, and a steep central gradient (Fig. 8h). The bar region also appears redder than the surroundings. The color map also shows clear hints of the existence of a small, red circumnuclear region.

Due to the large size of this galaxy, the bulge+disk decomposition is not reliable (Fig. 8f), as confirmed by the high residuals (Fig. 8g).

\subsection{NGC 4253 (UGC 7344, Mrk 766)}

This galaxy has a thick bar and a weak external ring (Fig. 9a). Its nucleus is displaced relatively to the centroid of the outer isophotes. The bar parameters from Fig. 9e are in agreement with previous results in $J$ (Alonso-Herrero et al. 1998) and $K$ (McLeod \& Rieke 1995; Peletier et al. 1999). Note that this direction is also that of the stellar bar seen at optical wavelengths (Mulchaey \& Wilson 1996; Mulchaey et al. 1996).

The sharp-divided image (Fig. 9b) reveals the presence of a small structure, possibly a secondary bar, roughly perpendicular to the main bar. This feature appears even more clearly on the difference image (Fig. 9c).

The $J / K^{\prime}$ image shows a double nuclear structure with a $\left(J-K^{\prime}\right)$ color index redder than the rest of the galaxy 
(Fig. 9d); this structure can explain the observed decentering. Notice that in the very central zone $(r<3$ arcsec) $\epsilon$ is not the same in $J$ and $K$ (Fig. 9e). This feature seems to correspond to that delineated by the dust pattern that surrounds the innermost 2 arcsec in the HST optical image (Malkan et al. 1998). Unfortunately, the nucleus is saturated in the infrared HST image, so we cannot analyse the presence of faint nuclear elongations. Nevertheless, a curved dust pattern feature with a radius of $\approx 4$ arcsec can be seen along the NE-SW direction (Fig. 30), favouring the presence of a nuclear bar.

The profile is in good agreement with that given by McLeod \& Rieke (1995). The bulge+disk fit is quite satisfactory (Figs. 9f and 9g).

\subsection{NGC 4507}

The $K^{\prime}$ image agrees with that of Mulchaey et al. (1997), and indeed shows no clear presence of a bar (Fig. 10a). A noticeable curved dust lane to the SE reaching the innermost 2 arcsec is visible in the HST image by Malkan et al. (1998).

The sharp-divided image (Fig. 10b) clearly shows a bar that is also apparent on the difference image (Fig. 10c). This bar had been already reported by Mulchaey et al. (1997) $\left(r=10.5 \operatorname{arcsec}, \mathrm{PA}=53^{\circ}, \epsilon=0.34\right)$.

$\epsilon$ and PA show strong variations at $18-20$ arcsec from the nucleus, where the spiral arms begin (Fig. 10e) and produce a small bump over the bulge+disk fit (Fig. 10f). The bar is also apparent as a small enhancement over this fit. However, due to the relative weakness of the bar and arms, the residuals remain small (Fig. 10g). The $J / K^{\prime}$ image is quite smooth except in the very nucleus, which has much redder colors (Fig. 10d) than the outer galaxy.

\subsection{NGC 4785}

This galaxy has a bright thick bar (Fig. 11a) and a complex spiral structure with several arms, including a thin arm to the south with several blobs. Note that the major axis orientation varies with radius in the central 14 arcsec (Fig. 11e).

The sharp-divided image reveals the presence of a ring and shows well the spiral arms (Fig. 11b). These features are also clearly visible in the difference image (Fig. 11c), where a small central bar seems to be present. The existence of such a small bar is confirmed by the $J / K$ image (Fig. 11d) and by the variations of $\epsilon$ and PA with radius (Fig. 11e) both in ground based and HST images. The sharp-dividing method applied to the NICMOS HST image shows an inner elongation of $\approx 1.5$ arcsec along $\mathrm{PA}=109^{\circ}$ (Fig. 31).

The colour image is quite constant except at the location of the arms and inner ring which appear redder in the $J / K^{\prime}$ color image (Figs. 11d and 11h).

\subsection{2. $N G C 5347$}

The $K^{\prime}$ image of this galaxy only shows a large bar, the rest of the structure being very smooth with hardly any hint for the presence of spiral arms (Fig. 12a), in agreement with Mulchaey et al. (1997). Even the sharp-divided image shows no structure (Fig. 12b) except for the bright nucleus.

However, the extremities of the bar appear well in the difference image (Fig. 12c), and the existence of a bar is confirmed by the PA and $\epsilon$ variations both from ground based and HST images (Fig. 12e) (in agreement with Mulchaey et al. 1997) and by the excess over the bulge+disk fit (Figs. $12 \mathrm{f}$ and $12 \mathrm{~g}$ ).

Surprisingly, the $J / K^{\prime}$ image reveals a double structure separated by $\approx 3$ arcsec, resembling a double nucleus (Fig. 12h). The close inspection of the color optical to near-infrared image by Regan \& Mulchaey (1999), shows that this may result from the dust lane crossing the nucleus.

\subsection{3. $N G C 5728$}

This galaxy is larger than the size of our infrared images, so we will only present here the properties of the inner regions.

The $K^{\prime}$ image clearly shows the presence of a small bar within the large bar (Fig. 13a), both already reported by Wozniak et al. (1995) in their BVRI images. The small bar appears even more strongly in the sharp-divided image (Fig. 13b), where a small inner ring is also visible $(r \approx 4$ arcsec, corresponding to the ring reported by Buta \& Crocker 1993). The small bar and ring as well as the extremities of the large bar are also visible in the difference image (Fig. 13c), while the nucleus and ring show weaker $J$ relative to $K^{\prime}$ than the rest of the galaxy (Fig. 13d).

Two maxima of $\epsilon$ coupled with a constant PA (Fig. 13e) allow us to determine both primary and secondary bar parameters (see also Shaw et al. 1993; Wozniak et al. 1995).

Since we obviously do not reach the galaxy disk, the bulge+ disk fit cannot be fully correct (Figs. 13f and 13g).

\subsection{ESO 139-12}

This galaxy shows a very regular structure in the $K^{\prime}$ band (Fig. 14a), with a luminous bulge and no bar. No structure appears either in the sharp-divided or difference images (Figs. 14b and 14c), and the $J / K^{\prime}$ image appears to be very smooth (Figs. 14d and h). A faint and quite curved dust lane is visible in the published HST image (Malkan et al. 1998) within the central 2 arcsec only.

While $\epsilon$ does not vary much with radius, the PA does vary strongly as the isophotes are seen to rotate (Fig. 14e), probably due to the effect of the flocculent spiral structure. 
The bulge+disk fit is acceptable, with residuals smaller than $20 \%$ throughout (Figs. $14 \mathrm{f}$ and $14 \mathrm{~g}$ ). The $J / K^{\prime}$ image shows a red nuclear region, also visible in the color gradient plot (Fig. 14h).

\subsection{NGC 6814}

This galaxy shows a beautiful spiral structure on the $K^{\prime}$ image, with spiral arms emerging from a thick bar (Fig. 15a). Our image appears to be quite similar to that of Mulchaey et al. (1997).

The spiral structure is seen in the sharp-divided image, where the bar is traced as a faint elongation (Fig. 15b). The spiral structure is clearly observed in the difference image (Fig. 15c).

Except for the very nucleus, the $J / K^{\prime}$ image remains constant throughout the galaxy, excepting the innermost 2 arcsec, which are redder (Figs. $15 \mathrm{~d}$ and $15 \mathrm{~h}$ ).

The bar parameters from (Fig. 15e) are in agreement with Mulchaey et al. (1997). Note the strong and sudden change of PA at the radius where the spiral arms begin. However the bulge+disk fit is very good (Figs. 15f and $15 \mathrm{~g})$. The NICMOS HST image is saturated in the nucleus, so it cannot be used to gather information on the presence of inner structures. The bar is seen as a thick elongated 10 arcsec structure along the NS direction in Fig. 32 .

\subsection{6. $N G C 6860$}

This galaxy has an asymmetrical bar along $\mathrm{PA} \sim 10^{\circ}$, more extended to the north than to the south (Fig. 16a). The ring is not apparent in the $K^{\prime}$ image, where we barely detect the beginning of a spiral arm north of the bar.

The bar in the sharp-divided image appears clearly bent (Fig. 16b), possibly due to the dynamical effects of a small inner bar (Figs. 16b, 16c, 16d). The difference image (Fig. 16c) also evidences a faint spiral arm detaching from the southern end of the outer bar to the north east, and a brighter, tighter arm starting at the west end of the inner bar to the South. The variations of $\epsilon$ and PA with radius (Fig. 16e) hints on the presence of a secondary bar.

The $J / K^{\prime}$ image confirms the existence of a small redder inner bar (Fig. 16d), that is also evidenced by the dust lane structure in the HST image by Malkan et al. (1998). The main bar and the beginning of the spiral arms appear as bumps in the bulge+disk fit (Figs. 16f and 16g). The color gradient is very steep in the central regions (Fig. 16h).

\subsection{7. $N G C 6890$}

Despite its classification as a non-barred galaxy, the $K^{\prime}$ image of NGC 6890 reveals a strong bar (Figs. 17a and e), together with the beginning of two spiral arms at the extremities of the bar, the northern arm being brighter than the southern one (Fig. 17b). These features are also apparent in the $K^{\prime}$ image by Mulchaey et al. (1997), but these authors give $\mathrm{PA}=179^{\circ}$ corresponding to a radius $r \approx$ 14 arcsec, where the PA strongly decreases. However, their plots of the PA and $\epsilon$ show that the bar actually extends up to about 6 arcsec with $\mathrm{PA} \approx 15^{\circ}$, in agreement with our values. We also note that, even considering the differences in the photometry (see Table 4) the isophotal levels differ by more than 6 magnitudes, those by Mulchaey et al. being extremely bright and most probably incorrect.

The difference image reveals a beautiful spiral structure as well as a possible inner ring $(10-15$ arcsec $)$ (Fig. 17c). This inner ring seems confirmed by the $J / K^{\prime}$ image (Fig. 17d), where $J$ appears to be somewhat larger around the nucleus than in the very central region. Notice a smooth decrease of $J$ relative to $K^{\prime}$ with increasing radius from 2 arcsec outwards (Fig. 17h). The NICMOS HST image shows spiraling structure reaching the central arsecond, with some hints of dust at 0.5 arcsec (Fig. 33).

The bulge+disk fit is quite good except in the arm regions (Figs. $17 \mathrm{f}$ and $17 \mathrm{~g}$ ).

\subsection{NGC 6951 (UGC 11604)}

This is the first galaxy of our sample for which all the necessary data has been acquired (imaging and spectroscopy); it has been studied in detail by Pérez et al. (1999) and will not be presented here. We just note that no secondary bar is detected even in NIR HST images.

\section{Description of individual non active galaxies}

\section{1. $N G C 151$}

This galaxy is larger than the size of our infrared images, so we only present here the properties of the inner regions.

The $K^{\prime}$ image shows a strong and broad bar, a disky structure, a large ring, and a nuclear region roughly perpendicular to the bar (Fig. 18a). Besides confirming the presence of a weak ring, the sharp-divided image reveals the existence of a small bar roughly perpendicular to the large bar (Fig. 18b). These features appear very clearly in the difference image (Fig. 18c) and are quantified by looking at the variations of $\epsilon$ and PA with radius (Fig. 18e). The Pa $\alpha$ image by Böker et al. (1999) shows that there is star formation ocurring at the ends of the bar and along the ring. The sharp-masking method applied to the HST F160W image confirms the presence of the inner bar which can be traced closer to the nucleus $(r \approx 2.5$ arcsec, $\mathrm{PA}=68^{\circ}$, Fig. 34).

The $J / K^{\prime}$ image shows a roughly circular redder region of 7 arcsec radius (Fig. 18d); the inner bar is seen as a slightly redder elongation along $\mathrm{PA} \approx 90^{\circ}$. 
The bulge+disk fits are merely indicative since the image is too small to reach the disk (Fig. 18f). They show the existence of the two bars.

\section{2. $I C 454(U G C 3570)$}

This galaxy has a particularly large bar, with the west arm more visible than the east one (Fig. 19a). The bright feature at the north west end of the bar is most probably a superimposed star. The central region of the bar and the west arm are clearly seen in the sharp-divided image (Fig. 19b), while the extremities of the bar appear more strongly on the difference image (Fig. 19c). This illustrates the fact that the sharp-dividing method is very well suited to detect elongated features which do not always appear as clearly in the difference images (in the cases where they can be nicely fit by ellipses). The PA and $\epsilon$ plots evidence the presence of a secondary bar (see also Fig. 19c).

The $J / K^{\prime}$ image and $\left(J-K^{\prime}\right)$ color gradient appear quite constant except in the bulge region and central bar where $\left(J-K^{\prime}\right)$ is redder (Figs. $19 \mathrm{~d}$ and $\left.19 \mathrm{~h}\right)$. The bulge+disk fit is quite good except at the extremities of the bar (Figs. 19f and 19g).

\section{3. $N G C 2712(U G C 4708)$}

This galaxy also has a very large bar, and well developed arms, the eastern being brighter than the western one (Fig. 20a). The sharp-divided image (Fig. 20b) reveals the presence of a small bar within the large bar. This is confirmed by the difference image (Fig. 20c), where the spiral arms are also apparent. The $\epsilon$ and PA variations allow to quantify the parameters for both bars (Figs. 20e and 20h).

The $J / K^{\prime}$ image shows that the small bar is bluer, at variance with the other nuclear regions which all appear redder in $\left(J-K^{\prime}\right)$ (Fig. 20e), including the surrounding emission. The joint analysis with the optical data will allow a full interpretation of this result. This structure is also visible in the color gradient, which remains constant further out (Fig. 20h).

The bulge+disk fit is obviously not good because of the strong bar and spiral arms (Figs. 20f and 20g).

\subsection{NGC 2811}

The entire galaxy almost fits in the $K^{\prime}$ image, which has a quite regular aspect, with no obvious bar or arms (Fig. 21a). This image is very similar to that by Jungwiert et al. (1997) in the $H$ band. On the other hand, the sharpdivided and difference images clearly reveal the presence of a bar, a smaller bar and weak spiral arms (Figs. 21b and 21c). The existence of two bars is confirmed by the variations of $\epsilon$ and PA (Fig. 21e).
The $J / K^{\prime}$ image is smooth, with a reddish bar and a blue surrounding ring (Fig. 21d). The bulge+disk fits are satisfactory, with small residuals throughout (Figs. 21f and $21 \mathrm{~g})$.

\section{5. $N G C 3571$}

This galaxy does not fit entirely in the $K^{\prime}$ frame. The $K^{\prime}$ image shows no evidence for a bar (Fig. 22a), but the sharp-divided image does show the existence of a small thick bar (Fig. 22b). Due to its small size and relative roundness, the bar cannot be detected either in the difference image (Fig. 22c) nor in the $\epsilon$ and PA plots (Fig. 22e). However, the ellipse fitting to the HST image evidences this structure as a clear elongation of $\sim 2$ arcsec along $\mathrm{PA}=90^{\circ}$ (Fig. 22e). This is confirmed by the HST sharpdivided image (Fig. 35) derived from the Böker et al. (1999) data.

The $J / K^{\prime}$ image is quite smooth, with the central region somewhat redder than the outer zones (Fig. 22d).

The bulge+disk fits are quite good (Figs. 22e and 22f) even if we do not have an image of the entire galaxy (Fig. 23h).

\section{6. $N G C 3835$ (UGC 6703)}

A thick bar and two spiral arms can be seen in the $K^{\prime}$ image (Fig. 23a) and even better in the sharp-divided image (Fig. 23b), while the difference image only shows the spiral arms clearly, but not the bar (Fig. 23c).

The characteristics of the bar can be seen in the variations of $\epsilon$ and PA with radius (Fig. 23e).

The $J / K^{\prime}$ image shows a small extension $(4 \operatorname{arcsec}$ in radius) along the east-west direction where the $\left(J-K^{\prime}\right)$ colour is bluer than for the rest of the galaxy (Fig. 23d). Better resolution images should be obtained in order to ascertain what causes the misalignment between this structure in the color image and the inner bar.

Because of the presence of the spiral arms, the bulge+disk fits are not very good (Figs. 23f and 23g).

\section{7. $N G C$ 4162 (UGC 7193)}

The $K^{\prime}$ image of this galaxy is quite irregular (Fig. 24a); two arms with a flocculent appearance can be seen, but no bar. The sharp-divided image does not show any strong feature (Fig. 24b). On the other hand, the difference image reveals a beautiful spiral structure (Fig. 24c), with a circumnuclear ring, three rather closed spiral arms starting from the ring towards the north and three other more open spiral arms also starting from the ring but towards the south (Fig. 24c).

The $\epsilon$ and PA variations with radius may reveal the presence of a bar (Fig. 24e); however, this feature does 
not appear very clearly in these plots and we only have some hints of a small thick bar in Fig. 24b, so we cannot be completely certain of its detection.

The bar and spiral arms appear in the bulge+disk fits in $J$ and $K^{\prime}$ as bumps at about 12, 20 and 38 arcsec (Figs. 24f and $24 \mathrm{~g}$ ). The $J / K^{\prime}$ image is quite smooth, with $\left(J-K^{\prime}\right)$ somewhat redder at the center (Fig. 24d).

\subsection{NGC 4290 (UGC 7402)}

The $K^{\prime}$ image shows the bar, spiral arms and a large weak external ring (Fig. 25a); the central isophotes are twisted relative to the bar. The sharp-divided image seems to show a small structure in the center at $\mathrm{PA}=90^{\circ}$ (Fig. 25b); the bar and spiral arms are clearly seen. These structures appear even more strongly in the difference image (Fig. 25c). The $\epsilon$ and PA variations are also consistent with the existence of two bars (Fig. 25e); however, it is difficult to say if the small structure in the center is really a small bar within the bar because it is thick and faint.

The bulge+disk fits are good except in the bar and spiral arm regions (Figs. 25f and $25 \mathrm{~g}$ ). The $J / K^{\prime}$ image and the color gradient are very smooth, with a slightly redder nucleus (Figs. 25d and 25h).

\subsection{NGC 4779 (UGC 8022)}

A mosaic of images was obtained for this galaxy, so our data really encompasses the entire object.

The $K^{\prime}$ image shows a strong bar, and weak flocculent and asymmetric spiral structure (Fig. 26a), as confirmed by the sharp-divided image (Fig. 26b). The bar and beginning of the spiral arms appear more clearly on the difference image (Fig. 26c). Chapelon et al. (1999) find a bar with $\mathrm{PA}=5^{\circ}$ and $r \approx 16$ arcsec. The bar that we measure is somewhat longer (Fig. 26e), in agreement with Friedli et al.'s (1996) result that bars are generally longer in $K$ than in $R$ (see also NGC 3660).

The $J / K^{\prime}$ colour image is fairly smooth (Fig. 26d). The bulge+disk fits clearly show the bar and spiral arm regions (Figs. 26f and 26g).

\subsection{NGC 6012 (UGC 10083)}

The $K^{\prime}$ image shows a bar with somewhat peculiar sharp edges; no evidence either for spiral arms or for a ring is seen, in spite of the classification of this galaxy as RSBR2* (Fig. 27a). This image is consistent with the smaller one obtained by de Jong \& van der Kruit (1994). The peculiar aspect of the bar is confirmed by the sharp-divided and difference images (Figs. 27b and 27c), where the bar seems to be crossed by a dust lane. In this case, the bar would be young and star formation should be observable along it; we will therefore look for evidence for star formation in our spectra.

The difference image also shows evidence for bright spots towards the edges of the bar, specially towards the north (Fig. 27c); although this could be a star superimposed on the galaxy, the fact that there is a faint southern counterpart tends to indicate that these regions may both be in the galaxy.

The variations of $\epsilon$ and PA with radius (Fig. 27e) give bar parameters in agreement with de Jong's (1996) results.

The bulge+disk model fits the profiles very nicely except in the region of the bar and bright spots (Figs. $27 \mathrm{f}$ and $27 \mathrm{~g}$ ). The $J / K^{\prime}$ image and color gradient are quite smooth throughout the galaxy (Figs. 27d and 27h).

\subsection{NGC 6155 (UGC 10385)}

A small bar is visible on the $K^{\prime}$ image, together with a spiral arm starting northward and another one wrapped towards the south east (Fig. 28a). Note that the center is displaced relatively to the centroid of the outer isophotes.

The bar and north spiral arm appear faintly on the sharp-divided image (Fig. 28b) and much more clearly on the difference image (Fig. 28c). The variations of $\epsilon$ and PA with radius (Fig. 28e) show that the bar reaches a radius of $6 \operatorname{arcsec}\left(\mathrm{PA}=120^{\circ}\right)$. A larger bar may be present up to $15 \operatorname{arcsec}\left(\mathrm{PA}=160^{\circ}\right)$; however, we cannot be certain that it is a bar since the spiral arms seem to start at a smaller radial distance than the edges of this structure.

The bulge+disk model shows the strong contribution of the bar and spiral arms (Figs. $28 \mathrm{f}$ and $28 \mathrm{~g}$ ).

The $J / K^{\prime}$ image is fairly smooth and becomes redder at the very center (Figs. 28d and 28h).

\section{Summary}

We have observed a sample of 29 isolated spiral galaxies: 18 host an AGN (Seyfert 1 or Seyfert 2) and 11 are nonactive galaxies. We present here the infrared data in the $J$ and $K^{\prime}$ bands, as well as the image analysis. This includes sharp-divided images obtained by dividing the observed images by their filtered counterparts, difference images, obtained by fitting ellipses to the isophotes and subtracting such models to the observed images, and colour $J / K^{\prime}$ images. A bulge+disk model was fit to the image profiles, and the corresponding fit parameters are given.

Four (one) out of five (one) of the optically classified non-barred active (control) galaxies harbour a bar. Three of them had already been described as barred galaxies, as derived either from NIR (UGC 1395, which also has a secondary bar, and NGC 6890) or optical I (NGC 3281) analyses. The other two (NGC 2639 and NGC 4162) are classified as barred for the first time. For 15 (9 active, 6 control) out of 24 (14 active, 10 control) of 
the optically classified barred galaxies (SB or SX) we find that a secondary bar (or a disk, a lense or an elongated ring; see Table 5 for those cases for which the reported central elongation remains uncertain) is present.

A discussion on the physical properties of these galaxies, together with the comparison of the properties of active versus non-active galaxies derived from these data will be presented in a forthcoming paper (Márquez et al., in preparation).

Acknowledgements. I. Márquez acknowledges financial support from the Spanish Ministerio de Educación y Ciencia (EX94-8826734). This work is financed by DGICyT grants PB93-0139 and PR95-329. We acknowledge financial support from INSU-CNRS for several observing trips. Financial support to develop the present investigation has been obtained by the French-Spanish HF1996-0104 and HF1998-0052 and the Chilean-Spanish bilateral agreement CSIC-CONICYT 99CL0018.

We thank Ron Probst, who made available the SQIID package for the reduction of infrared images within IRAF available to us. This research has made use of the NASA/IPAC extragalactic database (NED), which is operated by the Jet Propulsion Laboratory under contract with the National Aeronautics and Space Administration.

\section{References}

Alonso-Herrero A., Ward M.J., Kotilainen J., 1996, MNRAS 278, 902

Alonso-Herrero A., Simpson C., Ward M.J., Wilson A.S., 1998, ApJ 495, 196

Böker T., Calzetti D., Sparks W., et al., 1999, ApJS 124, 95

Buta R., Crocker D.A., 1993, AJ 105, 1344

Chapelon S., Contini T., Davoust E., 1999, A\&A 345, 81

de Jong R.S, van der Kruit P.C., 1994, A\&AS 106, 451

de Jong R.S., 1996, A\&AS 118, 557

Erwin, P., Sparke L., 1999, ApJ 521, L37
Friedli D., Wozniak H., Martinet L., Bratschi P., 1996, A\&AS 118,461

Glass I.S., Moorwood A.F.M., 1985, MNRAS 214, 429

Heckman T.M., 1980, A\&A 87, 142

Hunt L.K., Malkan M.A., Rush B., et al., 1999, astro-ph/9905066

Jedrzejewski R.I., 1987, MNRAS 226, 747

Jungwiert B., Combes F., Axon D.J., 1997, A\&AS 125, 479

Laine S., Knapen J.H., Pérez-Ramírez D., Doyon R., Nadeau D., 1999, MNRAS 302, L33

Maiolino R., Rieke G.H., 1995, ApJ 454, 95

Malkan M.A., Gorjian V., Tam R., 1998, ApJS 117, 25

Márquez I., Moles M., 1996, A\&AS 120, 1

Márquez I., Moles M., Masegosa J., 1996, A\&A 310, 401

Márquez I., Moles M., 1999, A\&A 344, 421

McLeod K.K., Rieke G.H., 1995, ApJ 441, 96

Moles M., Márquez I., Pérez E., 1995, ApJ 438, 604

Moriondo G., Giovanardi C., Hunt L.K., 1998, A\&AS 130, 81

Mulchaey J.S., Wilson A.S., 1996, ApJ 455, L17

Mulchaey J.S., Wilson A.S., Tsvetanov Z., 1996, ApJS 102, 309

Mulchaey J.S., Regan M.W., 1997, ApJL 482, L135

Mulchaey J.S., Regan M.W., Kundu A., 1997, ApJS 110, 299

Peletier R.F., Knapen J.H., Shlosman I., et al., 1999, astro-ph/9905076

Pérez E., Márquez I., Marrero I., et al., 1999, A\&A (in press)

Regan M.W., Mulchaey J.S., 1999, AJ 117, 267

Shaw M.A., Combes F., Axon D.J., Wright G.S., 1993, A\&A 273,31

Shlosman I., Frank J., Begelman M., 1989, Nat 338, 45

Simkim S.M., Su H.J., Schwarz M.P., 1980, ApJ 237, 404

Sofue Y., Yoshida S., Aoki T., et al., 1994, PASJ 46, 1

Véron-Cetty M.P., Véron P., 1993, "A catalogue of Quasars and Active Nuclei", 6th Edition, ESO Scientific Report \#13

Wozniak H., Friedli D., Martinet L., Martin P., Bratschi P., 1995, A\&AS 111, 115

Xanthopoulos E., 1996, MNRAS 280, 6 